\title{
ON THE COMMUTATIVITY OF ULTRAPRODUCTS WITH DIRECT PRODUCTS
}

\author{
MICHEL HÉBERT
}

(Communicated by Andreas R. Blass)

\begin{abstract}
We show that an ultraproduct of direct products of structures is elementarily equivalent to a direct product (naturally defined over an ultraproduct of sets!) of ultraproducts of these structures.
\end{abstract}

It is a well-known fact of model theory that "finite products commute with ultraproducts": if $J$ is a finite set, $D$ is an ultrafilter on a set $I$, and $\left\{\mathfrak{U}_{i, j} \mid i \in I\right.$, $j \in J\}$ is a set of structures, then there is a canonical isomorphism:

$$
\Pi_{D}\left(\Pi_{J} \mathfrak{U}_{i, j}\right) \cong \Pi_{J}\left(\Pi_{D} \mathfrak{U}_{i, j}\right)
$$

(where $\Pi_{D} \mathfrak{B}_{i}$ is the $D$-ultraproduct of the $\mathfrak{B}_{i}$ 's and $\Pi_{J} \mathfrak{B}_{j}$ is the direct product of the $\mathfrak{B}_{j}$ 's; a more precise notation would be $\Pi_{D}\left(\Pi_{j \in J} \mathfrak{U}_{i, j} ; i \in I\right) \cong$ $\Pi_{j \in J}\left(\Pi_{D}\left(\mathfrak{U}_{i, j} ; i \in I\right)\right)$, but the context prevents any ambiguity). This can be checked directly (the isomorphism being the obviously defined one), or seen as a consequence of a very general algebraic property; namely, that "filtered colimits commute with finite limits" in a wide class of "natural" categories.

One readily finds counterexamples for infinite sets $J$. In fact, the commutativity cannot be extended to infinite $J$ even if the isomorphism is replaced by an elementary equivalence: let $I=J=\mathbb{N}$, and consider a statement $\phi$, which is true in a direct product $\Pi_{K} \mathfrak{B}_{k}$ if and only if it is true in each $\mathfrak{B}_{k}$ (for example $\phi=\forall x(R(x))$ for some relation symbol $R$ ), and a filter $D$ on $I$ containing all the cofinite subsets; then, if one chooses the structures $\mathfrak{U}_{i, j}$ such that $\mathfrak{U}_{i, j} \models \phi$ if and only if $i \geq j$, one gets $\Pi_{J}\left(\Pi_{D} \mathfrak{U}_{i, j}\right) \models \phi$ but $\Pi_{D}\left(\Pi_{J} \mathfrak{U}_{i, j}\right) \models \sim \phi$.

However, it seems to have been unnoticed before that there is a natural way to generalize to infinite sets $J$ the elementary equivalence above. One has to take on the right hand side the ultrapower (of sets) $\Pi_{D} J$ instead of $J$ (this really generalizes the finite case, as the natural embedding $J \rightarrow \Pi_{D} J$ an isomorphism when $J$ is finite): more explicitly, for each $x \in \Pi_{I} J=J^{I}$, denote by $[x]_{D}$ its equivalence class in $\Pi_{D} J$; then we will see that

$$
\Pi_{D}\left(\Pi_{J} \mathfrak{U}_{i, j}\right) \equiv \Pi_{[x]_{D} \in \Pi_{D} J}\left(\Pi_{D} \mathfrak{U}_{i, x(i)}\right) .
$$

Received by the editors April 5, 1989 and, in revised form, January 16, 1991.

1980 Mathematics Subject Classification (1985 Revision). Primary 03C20, 03 C40.

Key words and phrases. Ultraproduct. 
(One could find examples showing that replacing " $[x]_{D} \in \Pi_{D} J$ " by " $x \in \Pi_{I} J$ " invalidates the equivalence in general.) Furthermore, there is no necessity to keep $J$ fixed, and we show the following more general fact:

Theorem 1. Let $D$ be an ultrafilter on a set $I,\left\{J_{i}\right\}_{i \in I}$ be a family of disjoint sets, and $\left\{\mathfrak{U}_{j} \mid j \in \bigcup_{i \in I} J_{i}\right\}$ be a set of structures. Then

$$
\Pi_{D}\left(\Pi_{J_{i}} \mathfrak{U}_{j}\right) \equiv \Pi_{[x]_{D} \in \Pi_{D} J_{i}}\left(\Pi_{D} \mathfrak{U}_{x(i)}\right) .
$$

(As above, this is a short notation for

$$
\left.\Pi_{D}\left(\Pi_{j \in J_{i}} \mathfrak{U}_{j} ; i \in I\right) \equiv \Pi_{[x]_{D} \in \Pi_{D} J_{i}}\left(\Pi_{D}\left(\mathfrak{U}_{x(i)} ; i \in I\right)\right) .\right)
$$

The essence of the proof lies on a result due to Vaught ([5]; see also [2] for more details). Here is its formulation in [1] (where one can also find a proof):

Theorem 2. Given a sentence $\phi$, we can (effectively) find a number $n$ such that for all index sets $I$ and all structures $\mathfrak{U}_{i}, i \in I$, there is a subset $J$ of $I$ with at most $n$ elements such that for all $K, J \subset K \subset I$,

$$
\Pi_{i \in I} \mathfrak{U}_{i} \models \phi \quad \text { if and only if } \Pi_{i \in K} \mathfrak{U}_{i} \models \phi .
$$

Proof of Theorem 1. (a) We first assume that all cardinalities $\left|J_{i}\right|$ are infinite.

Let $\phi$ be a sentence such that $\Pi_{D}\left(\Pi_{J_{i}} \mathfrak{U}_{j}\right) \models \phi$, i.e. $\left\{i \in I \mid \Pi_{J_{i}} \mathfrak{U}_{j} \models \phi\right\} \in D$. By Theorem 2, there exist $m=m_{\phi} \in \mathbb{N}$ and $\left\{J_{i}^{\prime}\right\}_{I}$ where $J_{i}^{\prime} \subset J_{i},\left|J_{i}^{\prime}\right| \leq m$ for each $i \in I$, and such that for every $\left\{J_{i}^{\prime \prime}\right\}_{I}$ with $J_{i}^{\prime} \subset J_{i}^{\prime \prime} \subset J_{i}, i \in I$, we have $\Pi_{j \in J_{i}} \mathfrak{U}_{j} \models \phi$ if and only if $\Pi_{j \in J_{i}^{\prime \prime}} \mathfrak{U}_{j} \models \phi$.

Because all $\left|J_{i}\right|$ are infinite, we can find $\left\{J_{i}^{\prime \prime}\right\}_{i \in I}$ such that $\left|J_{i}^{\prime \prime}\right|$ is precisely $m$ for each $i \in I$, and such that $\left\{i \in I \mid \Pi_{J_{i}^{\prime \prime}} \mathfrak{U}_{j} \models \phi\right\} \in D$, i.e. $\Pi_{D}\left(\Pi_{J_{i}^{\prime \prime}} \mathfrak{U}_{j}\right) \models \phi$.

To clarify, we write $\Pi_{J_{i}^{\prime \prime}} \mathfrak{U}_{j}$ explicitly as $\mathfrak{U}_{j_{1}(i)} \times \cdots \times \mathfrak{U}_{j_{m}(i)}$. For each $k \in$ $\{1, \ldots, m\}$, let $x_{k}$ be the element of $\Pi_{I} J_{i}$ such that $x_{k}(i)=j_{k}(i)$ for every $i \in I$. Because $m$ is finite, we get

$$
\Pi_{D}\left(\Pi_{J_{i}^{\prime \prime}} \mathfrak{U}_{j}\right) \cong \Pi_{D}\left(\mathfrak{U}_{x_{1}(i)}\right) \times \cdots \times \Pi_{D}\left(\mathfrak{U}_{x_{m}(i)}\right) .
$$

Let $x^{\prime} \in \Pi_{I} J_{i}$ be such that $\left[x^{\prime}\right]_{D} \neq\left[x_{k}\right]_{D}$ for $k=1,2, \ldots, m$. Because all $\left|J_{i}\right|$ are infinite, we can suppose that $x^{\prime}(i) \neq x_{k}(i)$ for each $i \in I$ and each $k \in\{1,2, \ldots, m\}$. Now we have

$$
\Pi_{D}\left(\mathfrak{U}_{x_{1}(i)}\right) \times \cdots \times \Pi_{D}\left(\mathfrak{U}_{x_{m}(i)}\right) \times \Pi_{D}\left(\mathfrak{U}_{x^{\prime}(i)}\right) \cong \Pi_{D}\left(\mathfrak{U}_{x_{1}(i)} \times \cdots \times \mathfrak{U}_{x_{m}(i)} \times \mathfrak{U}_{x^{\prime}(i)}\right) .
$$

As for each $i \in I,\left\{x_{1}(i), \ldots, x_{m}(i), x^{\prime}(i)\right\}$ is a set of distinct elements of $J_{i}$ containing $J_{i}^{\prime \prime}$, one can see that $\Pi_{D}\left(\mathfrak{U}_{x_{1}(i)} \times \cdots \times \mathfrak{U}_{x_{m}(i)} \times \mathfrak{U}_{x^{\prime}(i)}\right) \vDash \phi$.

We can repeat the argument for any finite number of $x^{\prime}$, hence we have shown that:

For every finite set $\left\{\Pi_{D},\left(\mathfrak{U}_{x_{1}^{\prime}(i)}\right), \ldots, \Pi_{D}\left(\mathfrak{U}_{x_{n}^{\prime}(i)}\right)\right\}$ of ultraprod-

(+) ucts such that the $\left[x_{1}^{\prime}\right]_{D}, \ldots,\left[x_{n}^{\prime}\right]_{D}$ are distinct and distinct from each one of $\left[x_{1}\right]_{D}, \ldots,\left[x_{m}\right]_{D}$, we have $\Pi_{D}\left(\mathfrak{U}_{x_{1}(i)}\right) \times \cdots \times$ $\Pi_{D}\left(\mathfrak{U}_{x_{m}(i)}\right) \times \Pi_{D}\left(\mathfrak{U}_{x_{1}^{\prime}(i)}\right) \times \cdots \times \Pi_{D}\left(\mathfrak{U}_{x_{n}^{\prime}(i)}\right) \models \phi$. 
If we now suppose that $\Pi_{Y}\left(\Pi_{D}\left(\mathfrak{U}_{x(i)}\right)\right) \models \sim \phi$, for $Y=\Pi_{D} J_{i}$, then, by Theorem 2 again, there exists a finite subset $Y^{\prime}$ of $Y$ such that for every $Y^{\prime \prime} \subset Y$ disjoint from $Y^{\prime}$, we have $\Pi_{Y^{\prime \prime}}\left(\Pi_{D}\left(\mathfrak{U}_{x(i)}\right)\right) \times \Pi_{Y^{\prime}}\left(\Pi_{D}\left(\mathfrak{U}_{x(i)}\right)\right) \models \sim \phi$. This contradicts $(+)$ (take $Y^{\prime \prime}=\left\{\left[x_{1}\right]_{D}, \ldots,\left[x_{m}\right]_{D}\right\}$ ).

(b) For the general case, let $\alpha=\sup _{i \in I}\left(\left|J_{i}\right|, \omega\right)$. We add to each set $\left\{\mathfrak{U}_{j} \mid j \in J_{i}\right\}$ a sufficient number of "trivial" structures $\{*\}$ to get sets $J_{i}^{\prime}$ of cardinality $\alpha$. (A trivial structure is one with only one element $*$ and such that $R(*, *, \ldots, *)$ for every relation $R$.) Let us denote these new sets by $\left\{\mathfrak{U}_{j^{\prime}}^{\prime} \mid j^{\prime} \in J_{i}^{\prime}\right\}$, where $\mathfrak{U}_{j^{\prime}}^{\prime}=\{*\}$ if $j^{\prime} \in J_{i}^{\prime} \backslash J_{i}$ and $\mathfrak{U}_{j^{\prime}}^{\prime}=\mathfrak{U}_{j^{\prime}}$ otherwise. Clearly $\Pi_{D}\left(\Pi_{J_{i}} \mathfrak{U}_{j}\right) \cong \Pi_{D}\left(\Pi_{J_{i}^{\prime}} \mathfrak{U}_{j^{\prime}}^{\prime}\right)$.

Let us consider an ultraproduct $\Pi_{D}\left(\mathfrak{U}_{x^{\prime}(i)}^{\prime}\right)$, where $x^{\prime}(i) \in \Pi_{I} J_{i}^{\prime}$. We distinguish two cases:

(1) If $\left\{i \mid x^{\prime}(i) \in J_{i}\right\} \in D$, then $\Pi_{D}\left(\mathfrak{U}_{x^{\prime}(i)}^{\prime}\right) \cong \Pi_{D}\left(\mathfrak{U}_{x(i)}\right)$, where $x(i)=x^{\prime}(i)$ if $x^{\prime}(i) \in J_{i}$ and $x(i)$ is any element of $J_{i}$ if $x^{\prime}(i) \in J_{i}^{\prime} \backslash J_{i}$.

(2) If $\left\{i \mid x^{\prime}(i) \in J_{i}\right\} \notin D$, then $\Pi_{D}\left(\mathfrak{U}_{x^{\prime}(i)}^{\prime}\right) \cong\{*\}$.

From (1) and (2), we deduce that $\Pi_{\Pi_{D} J_{i}^{\prime}}\left(\Pi_{D} \mathfrak{U}_{x^{\prime}(i)}^{\prime}\right) \cong \Pi_{\Pi_{D} J_{i}}\left(\Pi_{D} \mathfrak{U}_{x(i)}\right)$. By part (a), we have $\Pi_{\Pi_{D} J_{i}^{\prime}}\left(\Pi_{D} \mathfrak{U}_{x^{\prime}(i)}^{\prime}\right) \equiv \Pi_{D}\left(\Pi_{J_{i}^{\prime}} \mathfrak{l}_{j}^{\prime}\right)$, and then the result.

As a corollary, one easily obtains the main result of [4], which states that if $K$ is a compact class of structures (i.e. $\{\mathfrak{U} \mid \mathfrak{U} \equiv \mathfrak{B}$ for some $\mathfrak{B} \in K\}$ is elementary), then $\mathbf{P}(K)$ (the class of all direct products of structures in $K$ ) is also compact. (The proof of Makkai, which is quite complicated, lies on a much refined version of Theorem 2 also found in [2]). This has interesting consequences on the strength of the preservation theorem for direct products (see [3]).

The referee pointed out to us that Theorem 1 follows more easily from an unpublished work of Y. Vourtsanis [6]. As in the refined version of Theorem 2 mentioned above (but in a different way), the main result of [6] associates to a given formula $\phi$ a finite set $\left\{\phi_{k}\right\}$ of formulas and then nicely characterizes the satisfaction of $\phi$ in a direct product in terms of the satisfaction of the $\phi_{k}$ 's in the factor.

\section{REFERENCES}

1. C. C. Chang and H. J. Keisler, Model theory, Springer-Verlag, New York, 1975.

2. S. Feferman and L. Vaught, The first order properties of products of algebraic systems., Fund. Math. 47 (1959), 57-103.

3. M. Hébert, Preservation and interpolation through binary relations between theories, $\mathrm{Z}$. Math. Logik Grundlag. Math. 35 (1989), 169-182. Corrections appear in 36 (1990).

4. M. Makkai, A compactness result concerning direct products of models, Fund. Math. 62 (1965), 313-325. 
5. R. L. Vaught, On sentences holding in direct products of relational systems, Proc. Internat. Congr. Math., vol. 2, Amsterdam, 1954.

6. Y. Vourtsanis, The structure of truth in products of structures, preprint, Bowling Green State Univ., 1988.

Département de Mathématiques et Statistique, Universite laval, Québec, P. Q. CANADA G1K 7P4 